\title{
Gastric variceal bleeding precipitated by a mycotic splenic arteriovenous fistula in a cirrhotic patient: radiological diagnosis and endovascular treatment
}

Than Naing $\underline{\text { Tun }}^{1}$, MMed, FRCR, Sundeep $\underline{\text { Punamiya }}^{1}$, MBBS, FAMS

\begin{abstract}
Splenic arteriovenous fistula (SAVF) is an unusual cause of portal hypertension, and is rarely associated with an infective aetiology. It is often difficult to identify SAVF clinically, and thus, radiological modalities are invariably required for diagnosis and treatment. We herein describe a case of SAVF occurring in a patient with compensated cirrhosis as a sequel to salmonella gastroenteritis, and presenting with acute gastric variceal bleeding. Selective transcatheter embolisation of the splenic artery was effective in controlling bleeding.
\end{abstract}

Keywords: embolisation, gastric varices, portal hypertension, salmonella infection, splenic arteriovenous fistula

\section{INTRODUCTION}

Splenic arteriovenous fistula (SAVF) is a rare but potentially treatable cause of portal hypertension. It usually occurs following the rupture of a splenic artery aneurysm, but can also be congenital, or secondary to trauma, surgery or infection..$^{(1,2)}$ The fistula rapidly elevates the portal venous pressure, resulting in symptoms such as variceal bleeding, ascites and abdominal pain. SAVF can be treated via transcatheter embolisation of the fistula, or splenectomy. We herein describe a rare case of SAVF secondary to Salmonella infection in a cirrhotic male patient who presented with an acute variceal bleeding. The patient was effectively treated with selective arterial embolisation.

\section{CASE REPORT}

A 29-year-old man was admitted to our hospital with high-grade fever, chills, diarrhoea and altered mental status secondary to Salmonella enteritis (SE). He had a significant past medical history of mental retardation, chronic anaemia, cardiomyopathy and compensated cryptogenic liver disease. At admission, his temperature was $38.6^{\circ} \mathrm{C}$, heart rate was 142 beats per min, blood pressure was $132 / 65 \mathrm{mmHg}$, and peripheral capillary oxygen saturation $\left(\mathrm{SpO}_{2}\right.$ ) was $100 \%$ on $2 \mathrm{~L}$ oxygen. The patient also appeared toxic, dehydrated and drowsy. His abdomen was soft and non-tender, and his liver and spleen were not palpable.

Laboratory test results were as follows: haemoglobin $9.1 \mathrm{gm} / \mathrm{dL}$; leucocyte count $31.3 \times 10^{9} / \mathrm{L}$; platelet count $345 \times 10^{9} / \mathrm{L}$; serum bilirubin $55 \mu \mathrm{mol} / \mathrm{L}$; serum albumin $36 \mathrm{mg} / \mathrm{dL}$; aspartate aminotransferase $73 \mathrm{U} / \mathrm{L}$; alanine aminotransferase $36 \mathrm{U} / \mathrm{L}$; alkaline phosphatase $217 \mathrm{U} / \mathrm{L}$; gamma-glutamyltransferase $92 \mathrm{U} / \mathrm{L}$; serum creatinine $57 \mu \mathrm{mol} / \mathrm{L}$; prothrombin time $16.7 \mathrm{~s}$; and international normalised ratio 1.35. Stool culture was positive for Salmonella group G bacilli, but negative for Clostridium difficile. Urine and blood cultures were negative. Upper gastrointestinal (UGI) endoscopy revealed nonspecific gastritis and a small Mallory-Weiss tear at the gastro-oesophageal junction. No oesophageal or gastric varices were noted. Colonoscopy showed patchy areas of inflammation, confirmed to be nonspecific on biopsy. The patient was started on intravenous (IV) ceftriaxone antibiotic therapy.

On Day 4 of hospitalisation, the patient developed massive rectal bleeding. His haemoglobin level dropped from $9.1 \mathrm{gm} / \mathrm{dL}$ to $6.7 \mathrm{gm} / \mathrm{dL}$ and was accompanied by hypotension. Proctoscopy revealed a large volume of fresh blood and blood clots. In view of the massive lower gastrointestinal bleeding and the unremarkable UGI endoscopy, urgent computed tomography (CT) angiography was performed. In the arterial phase, early, intense enhancement of the splenic and portal veins was observed, and in the venous phase, multiple portosystemic collateral vessels in the gastric and splenorenal regions were seen (Fig. 1). The findings were highly suggestive of SAVF and gastric varices. The liver was noted to be cirrhotic, and no splenic artery aneurysm, splenic enlargement or ascites was detected.

Selective coeliac and superior mesenteric catheter angiography was subsequently done, which confirmed an arteriovenous fistula (AVF) in the mid pole of the spleen, causing an early, rapid opacification of the splenic and portal veins (Fig. 2a). Retrograde flow within the posterior and left gastric veins was noted, opacifying the portosystemic collaterals in the gastric region (Fig. 2b). The splenic artery was of normal calibre, with no evidence of any splenic artery aneurysm. A 5-F angiographic catheter (Radifocus; Terumo Corporation, Tokyo, Japan) was advanced into the mid-splenic artery. Through this catheter, a 2.7-F microcatheter system (Progreat; Terumo Corporation, Tokyo, Japan) was manoeuvred into the segmental splenic branch supplying the SAVF (Fig. 2c). The segmental branch was embolised with a combination of 3-mm and 2-mm embolisation coils (VortX, Diamond-18 and Figure-8 Pushable Coils; Boston Scientific, Cork, Ireland) deployed distally, and 4-mm embolisation coils (MicroNester; Cook Medical, Bloomington, IN, USA) deployed proximally. Complete occlusion of the segmental artery was

1Department of Diagnostic Radiology, Tan Tock Seng Hospital, Singapore

Correspondence: Dr Than Naing Tun, Registrar, Department of Diagnostic Radiology, Tan Tock Seng Hospital, 11 Jalan Tan Tock Seng, Singapore 308433. drtntun@gmail.com 

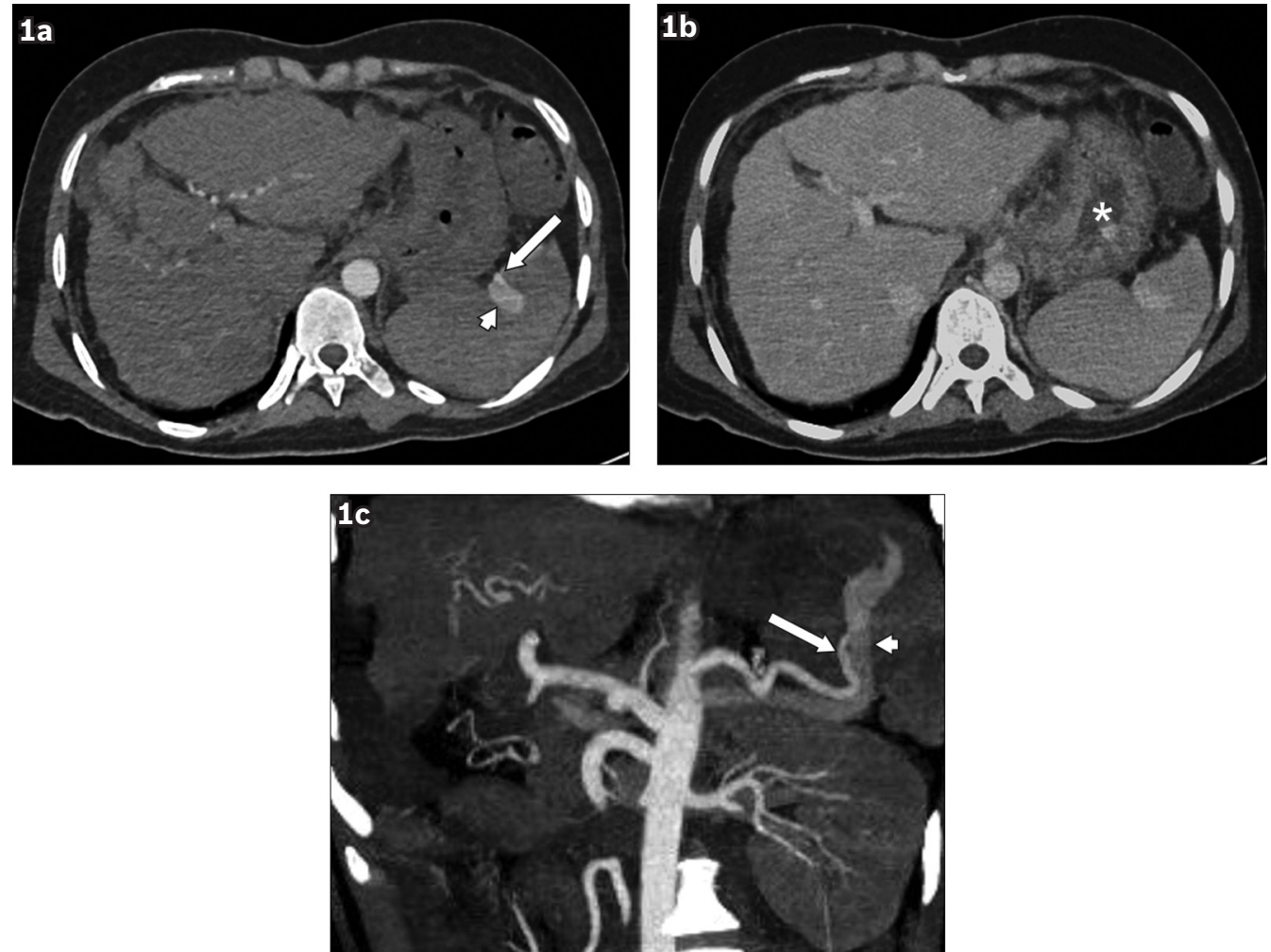

Fig. 1 CT angiograms of the abdomen show (a) the arteriovenous fistula between the segmental splenic artery (arrow) and the splenic vein (arrowhead) in the arterial phase; (b) varices in the gastric fundus $\left({ }^{*}\right)$ in the venous phase; and (c) early, intense enhancement of the splenic vein (arrowhead) and the splenic artery (arrow) devoid of aneurysm, in maximum intensity projection of the arterial phase.
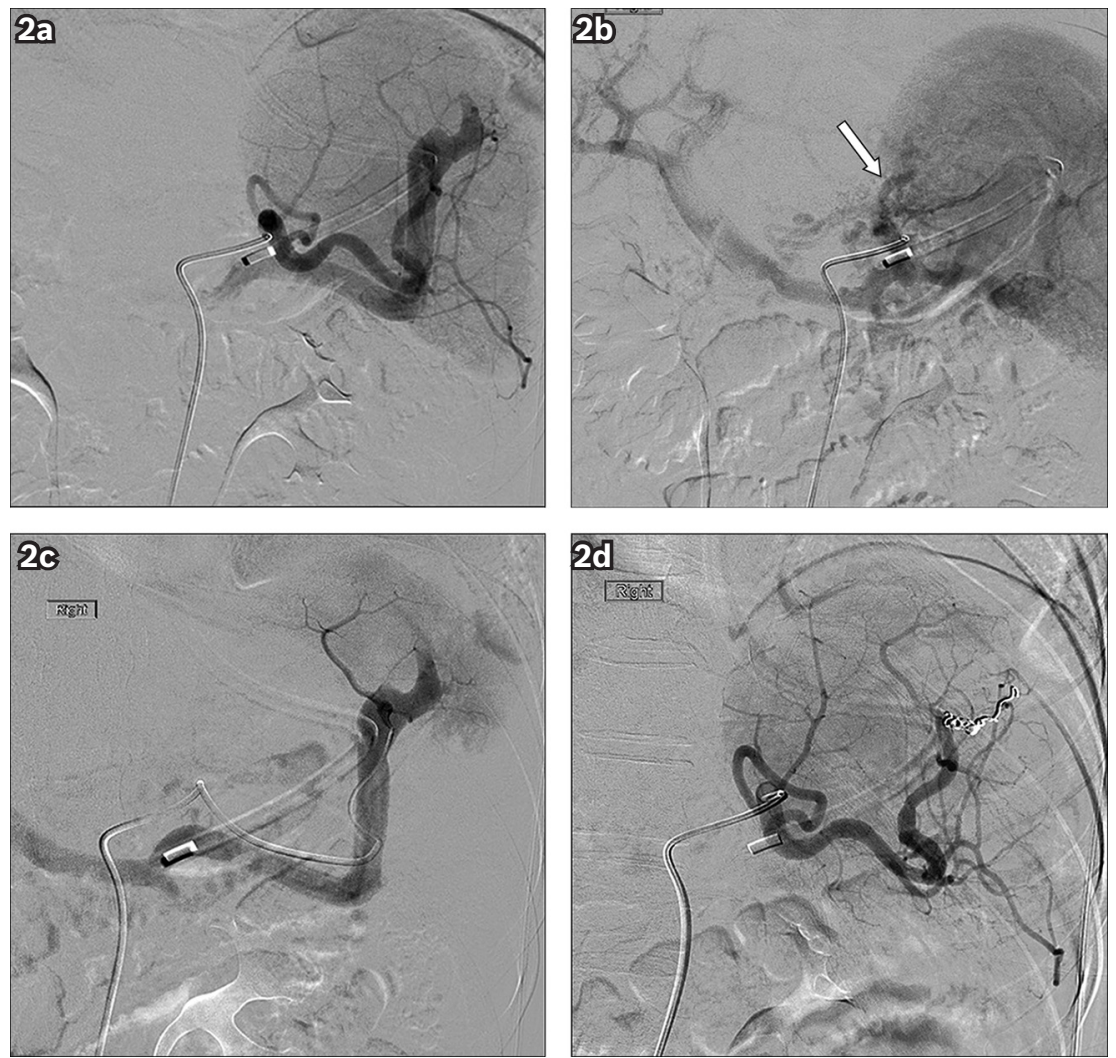

Fig. 2 Catheter splenic angiograms show (a) an arteriovenous fistula in the mid pole of the spleen, causing early and rapid opacification of the splenic vein in the arterial phase; (b) portosystemic collaterals via the prominent posterior gastric and left gastric veins (arrow); (c) the segmental splenic branch supplying the splenic arteriovenous fistula (SAVF), with immediate filling of the splenic vein; and (d) successful occlusion of the SAVF after embolisation coils were deployed into the feeding segmental splenic artery. 
achieved, with no subsequent filling of the SAVF (Fig. 2d), and the rest of the segmental splenic arteries remained patent.

Immediately following embolisation, repeat UGI endoscopy performed to confirm the gastric varices revealed a large blood clot in the fundus, with an underlying large, type I isolated gastric varix that was not seen in the endoscopy four days earlier. The patient had an uneventful recovery, with no further episodes of GI bleeding. He was discharged from hospital after 12 days on IV ceftriaxone and oral metronidazole, and was advised to undergo follow-up UGI endoscopy and wedged hepatic venous pressure tests. However, the patient's family declined any further evaluation. Nevertheless, he was well and symptom free at the six-month follow-up.

\section{DISCUSSION}

Portal hypertension represents an increase in hydrostatic pressure in the portal vein and its tributaries, and is classified as either pre-, intra- or post-hepatic, based on the anatomical location of its aetiology. SAVF, a rare cause of pre-hepatic portal hypertension, was first described by Wiegert in 1886 . Since then, approximately 100 cases have been reported in the literature, most of which were secondary to a rupture of the splenic artery aneurysm. ${ }^{(1)}$ Trauma, surgery and infection are other known causes of SAVF. Of these, a mycotic aetiology of SAVF is very rare, and to the best of our knowledge, none has been reported to have come from a Salmonella infection.

Salmonellas are Gram-negative anaerobic bacilli, of which Salmonella typhi and Salmonella paratyphi are the most well described species, as they cause enteric fever. Non-typhi salmonellosis, usually limited to the bowel and marked by gastroenteritis, has an extraintestinal spread in $5 \%-10 \%$ of patients, resulting in endovascular complications like endocarditis and arteritis, and may seed in and infect organs such as the liver, gallbladder, spleen, brain, pleura, bone and joints. ${ }^{(3)}$ Extraintestinal spread is more common in immunocompromised conditions such as acquired immunodeficiency syndrome, diabetes mellitus, malignancy, sickle-cell disease, rheumatologic disorders, and in patients on immunosuppression therapy. The diagnosis of SE is made on a positive stool culture, while that of the more morbid Salmonella bacteraemia is confirmed with a positive blood culture. However, $5 \%$ of bacteraemic patients may have a negative blood culture, which is usually seen with ongoing antibacterial therapy. ${ }^{(3)}$ The exclusion of other causes of SAVF and the temporal association of variceal bleeding following SE in our case had led us to conclude that SAVF was of mycotic origin, despite a negative blood culture.

An arterioportal fistula from any cause produces hyperkinetic syndrome due to the high pressure arterial inflow into the portal venous system. Sinusoidal fibrosis then develops, further elevating the portal vein pressure due to increased resistance. This phenomenon is more prominent if there is pre-existing chronic liver disease. The resultant portal hypertension usually manifests as bleeding gastric and oesophageal varices, upper abdominal pain, diarrhoea and/or ascites. ${ }^{(1,4)}$ Often difficult to diagnose clinically, SAVF may be suspected when a bruit is heard over the left flank; however, this is encountered in only $30 \%$ of cases. ${ }^{(4)}$ SAVF may also be suspected when features of portal hypertension are evident in the absence of liver disease, ${ }^{(5)}$ in patients with preexisting splenic artery aneurysms, and in patients with a history of surgery or trauma.

The diagnosis of SAVF can be made on Doppler, CT, magnetic resonance or catheter angiography. Typical Doppler findings include the following: (a) pulsatile, high-flow velocity within the fistula; (b) fairly pulsatile, high-velocity venous flow immediately distal to the fistula, sometimes extending into the splenic and portal veins; (c) increased afferent splenic arterial diameter and flow velocity; and (d) enlarged diameter and flow velocity in the splenic and portal veins. ${ }^{(6)}$ SAVF can be confirmed on CT angiography in the presence of dense opacification of the splenic vein in the arterial phase, along with varices and dilatation of the splenic vein. ${ }^{(7)}$ Nevertheless, catheter angiography is the gold standard for the detection of SAVF, and diagnosis is confirmed in the presence of early, rapid opacification of the splenic and portal veins, along with dilatation of the splenic artery and vein.

Our patient had well-compensated chronic liver disease of unknown aetiology. It is possible that he may have had preclinical portal hypertension that was significantly worsened by SAVF, thus leading to the early presentation of variceal bleeding. Unfortunately, the change in portal venous pressure before and after embolisation could not be confirmed with hepatic venous pressure gradient measurement, as this could have potentially delayed embolisation of the active haemorrhage.

The diagnosis of SAVF could be easily overlooked in the background of chronic liver disease. It was fortuitous that our patient underwent CT angiography to investigate the source and cause of his Gl bleeding, even though a previous endoscopic examination had been unremarkable. Without imaging, the gastric varices may have been presumed to be secondary to cirrhosis, and treated with traditional methods such as endoscopic glue injection, transjugular intrahepatic portosystemic shunt (TIPS), balloon-occluded retrograde transvenous obliteration of varices, or gastric devascularisation surgery. Given the high flow nature of SAVF, glue injection would likely have failed and/or been complicated by portal vein embolisation. TIPS has also been proven to be ineffective in the control of variceal bleeding in such patients. ${ }^{(8)}$

SAVF can be treated using transcatheter embolisation or surgery. ${ }^{(9)}$ Embolisation is highly effective and preferred over surgery, due to its lower morbidity and potential for partial splenic preservation. ${ }^{(4,8,10)}$ Surgery in the form of arterial ligation or splenectomy is usually reserved for patients who have failed transcatheter embolisation. Various materials can be used for embolisation, the choice of which is dependent on the location and flow characteristics of the fistula. In a high-flow AVF, particulate material (e.g. polyvinyl alcohol particles, trisacryl gelatin microspheres) and sclerosants (e.g. ethanol, sotradecol, ethanolamine oleate) are not the preferred embolic agents, as they flow easily through the fistula and get washed away, resulting in failure of occlusion and nontarget embolisation of portal venous branches. Embolisation coils and vascular occlusion plugs are 
the ideal agents in such cases. Cyanoacrylate glue and ethylene vinyl alcohol (Onyx) are infrequently used.

Splenic embolisation carries the risk of splenic infarction and other related problems such as splenic abscess, splenic rupture and pneumococcal sepsis, and the risk is potentially higher with complete splenic ablation than with segmental ablation. ${ }^{(11)}$ Our patient had an intra-parenchymal AVF that was supplied by a single segmental artery. Sub-selective embolisation of this single branch produced limited splenic infarction, thus minimising the risk of splenic embolisation.

In conclusion, mycotic SAVF is a rare cause of variceal bleeding, and the diagnosis could be overlooked in the background of chronic liver disease. It should be suspected when bleeding varices suddenly occur in patients with sepsis, and the suspicion should be confirmed with the appropriate imaging technique. Transcatheter embolisation is a safe and effective method of treatment for this complication.

\section{REFERENCES}

1. Di Lecce $F$, Viganò $P$, Busani $M$, et al. [Splenic artero-venous fistula and portal hypertension. A case report with review of the literature]. G Chir 2006; 27:328-30. Italian.

2. Quintiliani R, Ganguli P. Splenic arteriovenous fistula with bacterial endarteritis and endocarditis. JAMA 1970; 214:727-30.
3. Pegues DA, Miller SI. Salmonella species, including Salmonella typhi. In: Mandell GL, Bennett JE, Dolin R, eds. Mandell, Douglas, and Bennett's Principles and Practice of Infectious Diseases. 7th ed. New York: Elsevier, 2009: 2636-50.

4. Hung CF, Tseng JH, Lui KW, et al. Intractable oesophageal variceal bleeding caused by splenic arteriovenous fistula: treatment by transcatheter arterial embolization. Postgrad Med J 1999; 75:355-7.

5. Piscaglia F, Valgimigli M, Serra C, et al. Duplex Doppler findings in splenic arteriovenous fistula. J Clin Ultrasound 1998; 26:103-5.

6. Crusco F, Antoniella A, Puligheddu C, Fabbri AZ, Giovagnoni A. Postoperative splenic arteriovenous fistula detected with 16-multidetector computed tomography. Br J Radiol 2007; 80:e216-8.

7. Azar CR, Al-Kutoubi AO, Mourad FH. A short case of a splenic arteriovenous fistula coexisting with portal hypertension secondary to hepatitis C: Radiologic diagnosis and treatment. J Med Imaging Radiat Oncol 2010; 54:134-6.

8. Siablis D, Papathanassiou ZG, Karnabatidis D, et al. Splenic arteriovenous fistula and sudden onset of portal hypertension as complications of a ruptured splenic artery aneurysm: successful treatment with transcatheter arterial embolization. A case study and review of the literature. World J Gastroenterol 2006; 12:4264-6.

9. Madsen MA, Frevert S, Madsen PL, Eiberg JP. Splenic arteriovenous fistula treated with percutaneous transarterial embolization. Eur J Vasc Endovasc Surg 2008; 36:562-4.

10. Maloo MK, Burrows PE, Shamberger RC. Traumatic splenic arteriovenous fistula: splenic conservation by embolization. J Trauma 1999; 47:173-5.

11. Madoff DC, Denys A, Wallace MJ, et al. Splenic arterial interventions: anatomy, indications, technical considerations, and potential complications. Radiographics 2005; 25 Suppl 1:S191-211. 